\title{
Structure of Iron(III)-Tiron Complexes in Aqueous Solution
}

\author{
Kazuhiko Ozutsumi, Yoshihiko Uchima and Takuji KaWashima \\ Laboratory of Analytical Chemistry, Department of Chemistry, University of Tsukuba, \\ Tsukuba 305, Japan
}

\begin{abstract}
The structure of iron(III) complexes with 1,2-dihydroxybenzene-3,5-disulfonate (tiron ${ }^{4-}$ ) ion in aqueous solution has been studied by means of extended X-ray absorption fine structure (EXAFS) spectroscopy. EXAFS analyses of solutions containing $[\mathrm{Fe} \text { (tiron) }]^{-},\left[\mathrm{Fe}(\text { tiron })_{2}\right]^{]^{-}}$and $\left[\mathrm{Fe}(\text { tiron })_{3}\right]^{9-}$ complexes as the main species, respectively, revealed that the complexes have an octahedral structure. The $\mathrm{Fe}-\mathrm{O}$ bond lengths within the $\left[\mathrm{Fe}\left(\mathrm{H}_{2} \mathrm{O}\right)_{6}\right]^{3+},\left[\mathrm{Fe}(\mathrm{tiron})\left(\mathrm{H}_{2} \mathrm{O}\right)_{4}\right]^{-}$, $\left[\mathrm{Fe}(\text { tiron })_{2}\left(\mathrm{H}_{2} \mathrm{O}\right)_{2}\right]^{5-}$ and $\left[\mathrm{Fe}(\text { tiron })_{3}\right]^{9-}$ complexes are 202(1), 205(1), 202(1) and 204(1) pm, respectively, and are practically the same as that within the $\left[\mathrm{Fe}\left(\mathrm{H}_{2} \mathrm{O}\right)_{6}\right]^{3+}$ ion $(200 \mathrm{pm})$ determined by the X-ray diffraction method. The $\mathrm{Fe}-\mathrm{O}$ distances are thus practically the same for all the complexes, within experimental uncertainties. The similar distances may reflect the pure electrostatic interaction between iron(III) ion and oxygen atom in ligands.
\end{abstract}

Keywords Extended X-ray absorption fine structure, iron(III)-tiron, structure

1,2-Dihydroxybenzene-3,5-disulfonic acid ( $\mathrm{H}_{4}$ tiron) is a well-known reagent for determining titanium(III) or iron(III) ion photometrically. ${ }^{1}$ It is also known that the catalytic behavior of some metal ions on the redox reactions is well pronounced in the presence of tiron..$^{2,3}$ It is thought that this effect results from the complexation of tiron $^{4-}$ with metal ions. The complexation reaction of tiron ${ }^{4-}$ with metal ions has been investigated as well as that of catecholate ion ${ }^{4}$, both complexation reactions being rather similar. However, no information on the structure of metal complexes with these ligands in solution has been given. Since the solubility of the tiron complexes is higher than that of catecholato ones, the former are suitable for structure determinations in solution.

Siderophore found in microorganisms participates in the transfer and uptake of iron(III) ion. The large stability and the selectivity of the siderophore complexes enable them to uptake iron(III) ion of low concentrations in biological systems. ${ }^{5}$ Enterobactin, which is one of important siderophores, combines with iron(III) ion at the catechol groups. Catechol derivatives are thus good model compounds for siderophores.

The structure of iron(III) complexes with catechol derivatives has never been investigated in solution in spite of their analytical and biochemical importance. According to potentiometric and spectrophotometric measurements, a series of mononuclear [Fe(tiron)]', $\left[\mathrm{Fe}(\text { tiron })_{2}\right]^{5-}$ and $\left[\mathrm{Fe}(\text { tiron })_{3}\right]^{9-}$ complexes is formed in aqueous solution. ${ }^{1,5-9}$ The structure of the complexes has not been reported either in solution or in the solid state. In the present study, therefore, we aimed at determining the structure of tiron complexes with iron(III) ion in aqueous solution. The extented X-ray absorption fine structure (EXAFS) spectroscopy, which gives information on the local structure around an $\mathrm{X}$-ray absorbing atom, was used for the structure determination, because of the complex structure of tiron ${ }^{4-}$ ion with an aromatic benzene ring. Spectrophotometric measurements were also carried out to obtain the information on the individual complexes present in sample solutions.

\section{Experimental}

\section{Reagents}

All chemicals used were of reagent grade. Iron(III) perchlorate was recrystallized once from water. Iron(III) nitrate, Tiron $\left(\mathrm{Na}_{2} \mathrm{H}_{2}\right.$ tiron), sodium perchlorate and sodium hydroxide were used without further purification.

\section{Preparation of solutions}

Four test solutions were prepared for EXAFS measurements. Solution A was an aqueous iron(III) perchlorate solution involving a large amount of perchloric acid, which contains $\left[\mathrm{Fe}\left(\mathrm{H}_{2} \mathrm{O}\right)_{6}\right]^{3+}$ of known structure. ${ }^{10}$ Solutions B, C and D were prepared by dissolving iron(III) nitrate and Tiron, which had been mixed with stoichiometric amounts of sodium hydroxide, in water so that the $C_{\text {tiron }} / C_{\mathrm{Fe}(I I I)}$ mole ratios are one, two and three, respectively. According to the stability constants of iron(III)-tiron complexes so far reported ${ }^{4}$, the mono-, bis- and tris(tiron) complexes are expected to be present as the main species in solutions $\mathrm{B}, \mathrm{C}$ and $\mathrm{D}$, respectively. Concentrations of iron(III) ion, $C_{\mathrm{Fe}}$, in test solutions were photometrically deter- 
Table 1 The composition $\left(\mathrm{mol} \mathrm{dm}^{-3}\right)$ of sample solutions for EXAFS measurements

\begin{tabular}{ccc}
\hline Solution & $C_{\mathrm{Fe}} / \mathrm{mol} \mathrm{dm}^{-3}$ & $C_{\text {tiron }} / C_{\mathrm{Fe}}$ \\
\hline $\mathrm{A}$ & 1.00 & 0 \\
$\mathrm{~B}$ & 0.758 & 1.00 \\
$\mathrm{C}$ & 0.967 & 2.00 \\
$\mathrm{D}$ & 0.767 & 3.00 \\
\hline
\end{tabular}

mined as tris(phenanthroline)iron(II). Concentrations of tiron ${ }^{4-}$ ion, $C_{\text {tiron, }}$ were estimated from the weighted amounts of the compound. The compositions of the solutions are given in Table 1.

\section{EXAFS measurements}

EXAFS spectra were measured on a laboratory Xray absorption spectrometer consisting of a Johansson cut bent crystal $(\mathrm{Ge}(220))$ and a rotating anode generator (Rigaku Ru-200) at the Institute for Molecular Science. Details of the spectrometer have been described elsewhere. ${ }^{11}$

A pieced filter paper was immersed into sample solutions and then sealed in a polyethylene bag in order to prevent any evaporation of water. An effective jump at the absorption edge $(\mu x=1-2$ with the absorption coefficient $\mu$ and the sample thickness $x$ ) was obtained by changing the number of pieces of filter paper.

The absorbance $\mu x$ is given as $\ln \left(I_{0} / D\right.$, where $I_{0}$ and $I$ are X-ray intensities without and with a sample, respectively. The intensities $I_{0}$ and $I$ were simultaneously measured by an ion chamber and an SSD, respectively, and more than $1 \times 10^{6}$ counts of $I$ were obtained at each data point. Measurements were repeated at least twice over the whole range in order to examine the reproducibility of the results.

\section{Analysis of EXAFS data}

Background absorption other than that for the $\mathrm{K}$ edge of iron atom was estimated by least-squares fitting Victoreen formula ${ }^{12}$ to the pre-edge and was subtracted from the total absorption by extrapolation. The smooth K-shell absorption $\mu_{0}$ due to an isolated atom was evaluated by fitting a smooth curve to the observed absorption spectrum using a sixth-order polynomial function.

The EXAFS pattern was then extracted and normalized by the following equation;

$$
\chi(k)=\left(\mu(k)-\mu_{0}(k)\right) / \mu_{0}(k),
$$

where $k$ is the photoelectron wave vector ejected and is given as $\left[2 m\left(E-E_{0}\right)\right]^{1 / 2} / h . E$ represents the energy of the incident X-rays and $E_{0}$ is the binding energy of a $\mathrm{K}$ shell electron. The EXAFS spectra weighted by $k^{2}$ are depicted in Fig. 1. The $k^{2} \chi(k)$ values are converted to the radial distribution $F(r)$ as

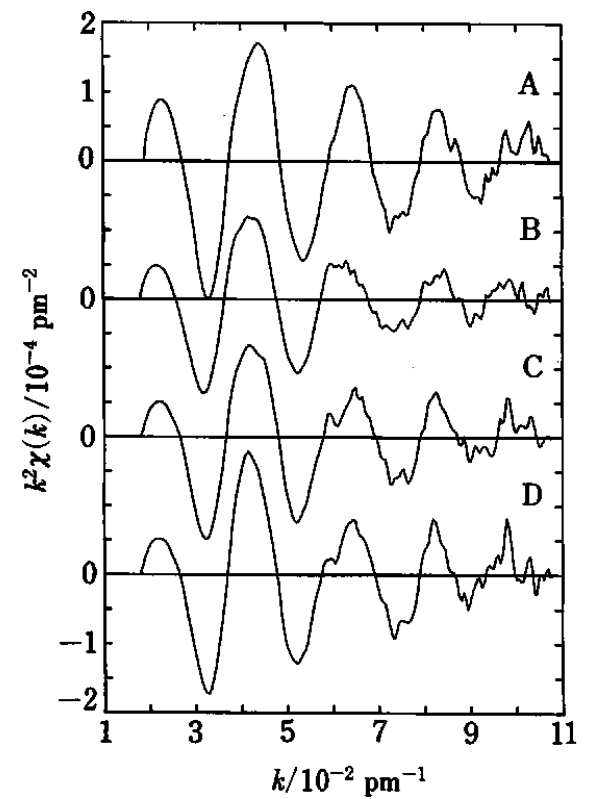

Fig. 1 The EXAFS spectra measured for sample solutions A through $D$.

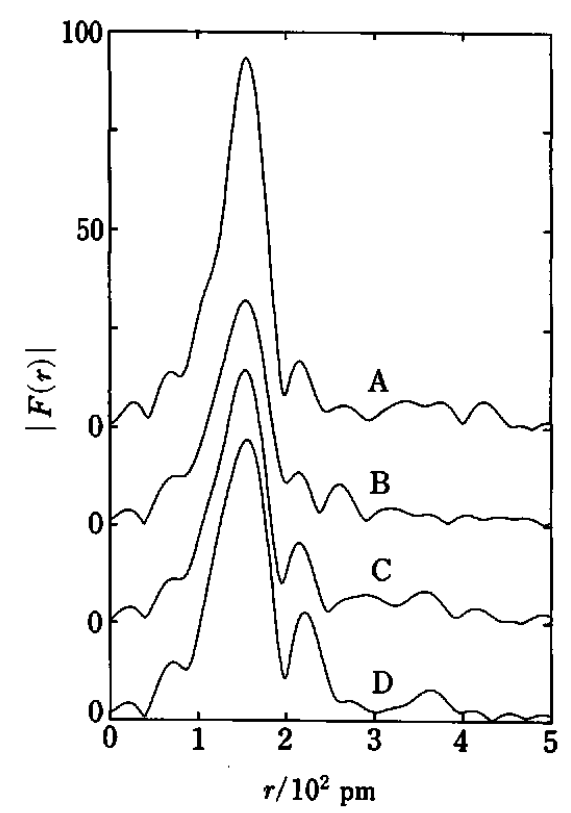

Fig. 2 The Fourier transforms $F(r)$ of the $k^{2} \chi(k)$ curves shown in Fig. 1, phase shift uncorrected.

$$
F(r)=(1 / 2 \pi)^{1 / 2} \int_{k_{\min }}^{k_{\max }} k^{2} \chi(k) W(k) \exp (-2 i k r) \mathrm{d} k
$$

$W(k)$ is the window function of the Hanning type. ${ }^{13}$ The $F(r)$ values are shown in Fig. 2.

A curve fitting procedure in the $k$-space for the refinements of structure parameters was applied to the Fourier filtered $k^{2} \chi(k)_{\text {obsd }}$ values to minimize the errorsquare $\operatorname{sum} U$ :

$$
U=\Sigma k^{4}\left(\chi(k)_{\mathrm{obsd}}-\chi(k)_{\mathrm{calcd}}\right)^{2} .
$$


The model function $\chi(k)_{\text {calcd }}$ is given by the single scattering theory as ${ }^{14-17}$

$$
\begin{aligned}
\chi(k)_{\text {calcd }}= & \Sigma\left[n_{j} /\left(k r_{j}^{2}\right)\right] \exp \left(-2 \sigma_{j}^{2} k^{2}-2 r_{j} / \lambda\right) F_{j}(\pi, k) \\
& \times \sin \left(2 k r_{j}+\alpha_{j}(k)\right)
\end{aligned}
$$

where $F_{j}(\pi, k)$ are the backscattering amplitudes from each of $n_{j}$ scatterers $j$ at distances $r_{j}$ from the X-ray absorbing atom. $\sigma_{j}^{2}$ is the mean square displacement of the equilibrium distance $r_{j}$ and $\lambda$ is the mean free path of the photoelectron ejected. $\alpha_{j}(k)$ is the total scattering phase shift experienced by the photoelectron. The values of $F_{j}(\pi, k)$ and $\alpha_{j}(k)$ in Eq. (4) were quoted from the tables reported by Teo and Lee. ${ }^{18}$ In the fitting procedures, the parameters $E_{0}$ and $\lambda$ were determined from the standard sample of known structure (an aqueous iron(III) perchlorate solution containing [Fe$\left.\left.\left(\mathrm{H}_{2} \mathrm{O}\right)_{6}\right]^{3+}\right)$ and then they were used as constants in the course of the structural analysis of unknown samples, while $r, \sigma$ and $n$ values were optimized as variables.

\section{Spectrophotometric measurements}

Electronic spectra were measured at $25^{\circ} \mathrm{C}$ by a UV2000 spectrophotometer (Shimadzu) equipped with a PC-9801VX computer (NEC), which recorded absorbance data in $1 \mathrm{~nm}$ intervals over the wavelength range $300-900 \mathrm{~nm}$. All test solutions contained $1 \mathrm{~mol} \mathrm{dm}^{-3}$ $\mathrm{NaClO}_{4}$ as an inert constant ionic medium. A flow cell with a path-length of $0.5 \mathrm{~cm}$ was connected to a titration vessel through Teflon and glass tubes. A solution containing iron(III) perchlorate and $\mathrm{H}_{2} \mathrm{Na}_{2}$ tiron was placed in a vessel and then titrated with a $0.1 \mathrm{~mol}$ $\mathrm{dm}^{-3} \mathrm{NaOH}$ solution. Spectrophotometric data at 40 selected wavelengths over the range $350-850 \mathrm{~nm}$ were employed for the least-squares calculation.

\section{Analysis of spectrophotometric data}

The overall formation of the $\left[\mathrm{Fe}_{o}(\text { tiron })_{p} \mathrm{H}_{q}\right]^{(30-4 p+q)+}$ complex (abbreviated as [opq]) can be defined as in Eqs. (5) and (6):

$$
\begin{aligned}
& o \mathrm{Fe}^{3+}+p \text { tiron }^{4-}+q \mathrm{H}^{+}=\left[\mathrm{Fe}_{o}(\text { tiron })_{p} \mathrm{H}_{q}\right]^{(30-4 p+q)+} \\
& \beta_{o p q}=\left[\mathrm{Fe}_{o}(\text { tiron })_{p} \mathrm{H}_{q}\right]^{(30-4 p+q)+} /\left[\mathrm{Fe}^{3+}\right]^{o}\left[\operatorname{tiron}^{4-}\right]^{p}\left[\mathrm{H}^{+}\right]^{q} .
\end{aligned}
$$

The absorbance measured in the solution $l$ at a given wavelength $\lambda_{m}$ is expressed by using the overall formation constants $\beta_{o p q}$ and the molar absorption coefficients $\varepsilon_{o p q}\left(\lambda_{m}\right)$ of $\left.\left[\mathrm{Fe}_{o} \text { (tiron) }\right)_{p} \mathrm{H}_{q}\right]^{(30-4 p+q)+}$ as Eq. (7).

$$
\begin{aligned}
A_{l m}= & \Sigma \Sigma \Sigma \varepsilon_{o p q}\left(\lambda_{m}\right) \beta_{o p q}\left[\mathrm{Fe}^{3+}\right]_{l}^{o}\left[\operatorname{tiron}^{4-}\right]_{l}^{p}\left[\mathrm{H}^{+}\right]_{l}^{q} \\
& +\varepsilon_{\mathrm{Fe}}\left(\lambda_{m}\right)\left[\mathrm{Fe}^{3+}\right]_{l}+\varepsilon_{\mathrm{tron}}\left(\lambda_{m}\right)\left[\text { tiron }^{4-}\right]_{l} .
\end{aligned}
$$

The concentrations of free $\mathrm{Fe}^{3+}$, tiron ${ }^{4-}$ and $\mathrm{H}^{+}$are related to their total concentrations, $C_{\mathrm{Fe}, l}, C_{\text {tiron, } l}$ and $C_{\mathrm{H}, l}$, in the solution $l$ by the mass-balance equations $(8)-(10)$, respectively.

$$
\begin{aligned}
C_{\mathrm{Fe}, l}= & {\left[\mathrm{Fe}^{3+}\right]_{l}+\Sigma \Sigma \Sigma o \beta_{o p q}\left[\mathrm{Fe}^{3+}\right]_{l}^{o}\left[\operatorname{tiron}^{4-}\right]_{l}^{p}\left[\mathrm{H}^{+}\right]_{l}^{q} } \\
C_{\text {tiron }, l} & =\left[\operatorname{tiron}^{4-}\right]_{l}+\Sigma \Sigma \Sigma p \beta_{o p q}\left[\mathrm{Fe}^{3+}\right]_{l}^{o}\left[\operatorname{tiron}^{4-}\right]_{l}^{p}\left[\mathrm{H}^{+}\right]_{l}^{q} \\
C_{\mathrm{H}, l}= & {\left[\mathrm{H}^{+}\right]_{l}+\Sigma \Sigma \Sigma q \beta_{o p q}\left[\mathrm{Fe}^{3+}\right]_{l}^{o}\left[\operatorname{tiron}^{4-}\right]_{l}^{p}\left[\mathrm{H}^{+}\right]_{l}^{q} } \\
& -K_{\mathrm{W}} /\left[\mathrm{H}^{+}\right]_{l^{*}}
\end{aligned}
$$

$K_{\mathrm{W}}$ represents the autoprotolysis constant of the solvent.

Molar absorption coefficients of complexes were determined by minimizing $\Sigma \Sigma\left(A_{l m, \text { obsd }}-A_{\text {lm,calcd }}\right)^{2}$ on the basis of the formation constants reported in the literature. ${ }^{8}$

\section{Results and Discussion}

\section{Spectrophotometry}

Figure 3 shows electronic specrtra measured with varying hydrogen ion concentrations at a given ligand to metal ion ratio in aqueous solution. Each spectrum should be represented as the sum of electronic spectra of individual species by taking into account the

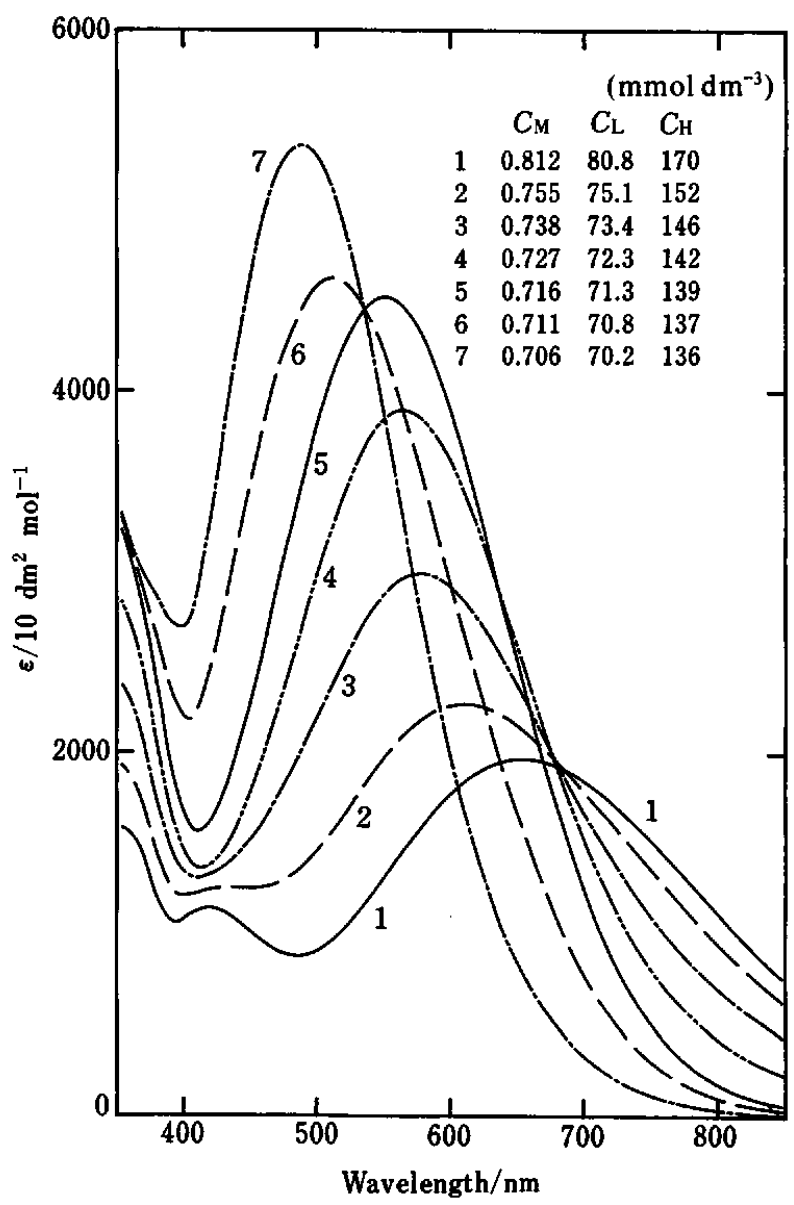

Fig. 3 Measured electronic spetra of Fe(III)-tiron solutions with varying hydrogen ion concentrations in the range $350-850 \mathrm{~nm}$. 
distribution of species in aqueous solution. The stability constants of the tiron complexes in various ionic media have been reported by several authors. ${ }^{4}$ Hence, we quoted the literatute values 8 in $1 \mathrm{~mol} \mathrm{dm}^{-3}$ $\mathrm{NaClO}_{4}$ and then analyzed the spectral changes on the basis of the stability constants.

The analysis was first performed by assuming the formation of the mono- ([110]), bis- ([120]) and tris(tiron) ([130]) complexes. As seen in Fig. 3, electronic spectra were also recorded at the high concentrations of hydrogen ion during the spectrophotometric measurements. The formation of the [111] complex was then also taken into consideration together with the [110], [120] and [130] complexes in the course of the analysis. However, the analytical result was not improved by assuming the formation of the [111] complex and, therefore, the formation of the [111] complex was not established under the experimental conditions in the present study.

Figure 4 depicts the molar absorption coefficients of individual iron(III)-tiron complexes extracted from measured spectral changes. The molar absorption coefficients of solutions for EXAFS measurements were also obtained by ten to twenty times dilution of the sample solutions and by using combination cells with a light path-length of $0.01 \mathrm{~cm}$. The coefficients obtained for sample solutions B, C and D satisfactorily agreed with those of mono-, bis- and tris(tiron) complexes shown in Fig. 4, respectively. Hence, the mono-, bisand tris(tiron) complexes are present as the predominant species in solutions $\mathrm{B}, \mathrm{C}$ and $\mathrm{D}$, respectively.

\section{EXAFS}

In Fig. 2, the Fourier transforms of the sample

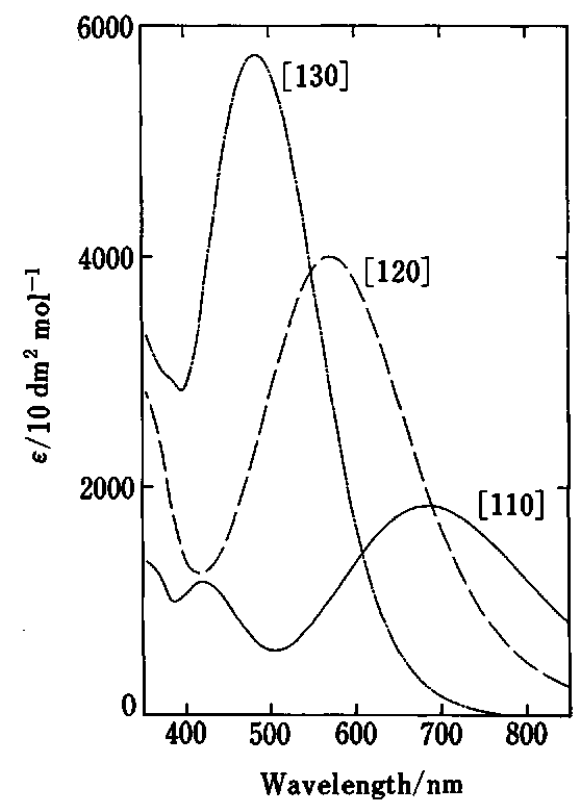

Fig. 4 Electronic spectra of individual iron(III)-tiron complexes in aqueous solution. The $\left[\mathrm{Fe}_{o}(\text { tiron })_{p} \mathrm{H}_{q}\right]^{(30-4 p+q)+}$ complex is represented as [opq]. solutions are depicted; here the first intense peaks around $150 \mathrm{pm}$ are ascribable to the bonds between iron(III) ion and oxygen atom in the first coordination sphere. The areas under the peaks of the Fourier transforms are very similar among solutions B, C and $D$ containing mono-, bis- and tris(tiron) complexes as a predominant species, respectively, suggesting that all tiron complexes have a similar structure in aqueous solution. The structure of the mono- and bis(tiron) complexes is thought to be octahedral, since the tris(tiron) complex is expected to have an six-coordinate octahedral structure. Thus, the mono-, bis- and tris(tiron) complexes are described as $\left[\mathrm{Fe}(\right.$ tiron $\left.)\left(\mathrm{H}_{2} \mathrm{O}\right)_{4}\right]$, $\left[\mathrm{Fe}(\text { tiron })_{2}\left(\mathrm{H}_{2} \mathrm{O}\right)_{2}\right]^{5-}$ and $\left[\mathrm{Fe}(\text { tiron })_{3}\right]^{9-}$, respectively.

The structure parameters were finally determined by a least-squares calculation applied to the Fourier filtered $k^{2} \chi(k)$ values over the range $4.5<k / 10^{-2} \mathrm{pm}^{-1}<$ 10.0. The $E_{0}$ and $\lambda$ values were first evaluated from an aqueous iron(III) perchlorate solution (solution A) involving hexaaquairon(III) ion with known structure and were found to be $7144.9(3) \mathrm{eV}$ and $592(11) \mathrm{pm}$, respectively. The $\mathrm{Fe}-\mathrm{O}$ bond length within the $\left[\mathrm{Fe}\left(\mathrm{H}_{2} \mathrm{O}\right)_{6}\right]^{3+}$ ion was also determined to be $202(1) \mathrm{pm}$, which agrees well with that reported by the X-ray diffraction method $(200 \mathrm{pm}){ }^{10}$ Therefore, the $E_{0}$ and $\lambda$ values were reasonably approximated by the present procedure.

The interatomic distances and Debye-Waller factors for the tiron complexes were then refined at the fixed coordination number of six (one-scatterer model) by adopting the $E_{0}$ and $\lambda$ values evaluated above. $\mathrm{Fe}-\mathrm{OH}_{2}$ and $\mathrm{Fe}-\mathrm{O}$ (tiron) bonds exist in the mono- and bis(tiron) complexes. Hence, in the course of the refinements, two kinds of the $\mathrm{Fe}-\mathrm{O}$ bonds were also taken into account for the mono- and bis(tiron) complexes (two-scatterer model). No reliable parameter values could, however, be obtained by the two-scatterer model owing to the strong correlation of parameters. Since the $\sigma$ values for the mono- and bis(tiron) complexes obtained by the one-scatterer model are not appreciably different from that for the tris(tiron) complex, the difference between two $\mathrm{Fe}-\mathrm{O}$ bond lengths should be very small. The results of the one-scatterer model are given in Table 2; the solid curves calculated by using the parameter values in the table well re-

Table 2 Results of the least-squares refinements of structure parameters for iron(III)-tiron complex in aqueous solution ${ }^{a}$

\begin{tabular}{lccrc}
\hline \multicolumn{1}{c}{ Complex } & Interaction & $r / p m$ & $\sigma / p m$ & $n$ \\
\hline$\left[\mathrm{Fe}\left(\mathrm{H}_{2} \mathrm{O}\right)_{6}\right]^{3+}$ & $\mathrm{Fe}-\mathrm{O}$ & $202(1)$ & $5.9(1)$ & $6^{\mathrm{b}}$ \\
{$\left[\mathrm{Fe}(\text { tiron })\left(\mathrm{H}_{2} \mathrm{O}\right)_{4}\right]^{-}$} & $\mathrm{Fe}-\mathrm{O}$ & $205(1)$ & $10.1(3)$ & $6^{\mathrm{b}}$ \\
{$\left[\mathrm{Fe}(\text { tiron })_{2}\left(\mathrm{H}_{2} \mathrm{O}\right)_{2}\right]^{5-}$} & $\mathrm{Fe}-\mathrm{O}$ & $202(1)$ & $9.1(1)$ & $6^{\mathrm{b}}$ \\
{$\left[\mathrm{Fe}(\text { tiron })_{3}\right]^{9-}$} & $\mathrm{Fe}-\mathrm{O}$ & $204(1)$ & $8.1(1)$ & $6^{\mathrm{b}}$ \\
\hline
\end{tabular}

Values in parentheses refer to standard deviations which were estimated from the results of repeated measurements. a. $E_{0}=7144.9(3) \mathrm{eV}$ and $\lambda=592(11) \mathrm{pm}$.

b. The values were kept constant during the calculations. 


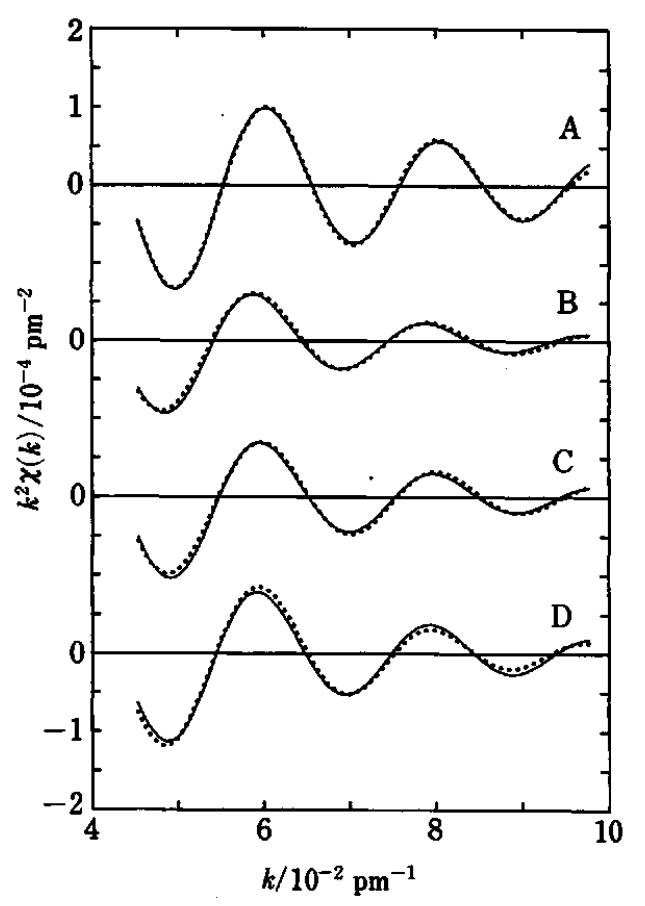

Fig. 5 The Fourier filtered $k^{2} x(k)$ curves of the main peak depicted in Fig. 2. The observed values are shown by dots and ones calculated using parameter values in Table 3 by solid lines.

produce the experimental points, as shown in Fig. 5.

The $\mathrm{Fe}-\mathrm{O}$ bond distances in $\left[\mathrm{Fe}\left(\mathrm{H}_{2} \mathrm{O}\right)_{6}\right]^{3+}$, [ $\mathrm{Fe}$ (tiron)$\left.\left(\mathrm{H}_{2} \mathrm{O}\right)_{4}\right]^{-},\left[\mathrm{Fe}(\text { tiron })_{2}\left(\mathrm{H}_{2} \mathrm{O}\right)_{2}\right]^{]^{-}-}$and $\left[\mathrm{Fe}(\text { tiron })_{3}\right]^{9-}$ are all $200 \mathrm{pm}$, within experimental uncertainties. According to the X-ray diffraction study on the structure of $\left[\mathrm{Fe}(\mathrm{ox})_{3}\right]^{3-}\left(\mathrm{ox}^{2-}:\right.$ oxalate ion) in aqueous solution ${ }^{19}$, the $\mathrm{Fe}-\mathrm{O}$ bond length within the complex was found to be $200 \mathrm{pm}$. The similar $\mathrm{Fe}-\mathrm{O}$ bond distances may be due to the pure electrostatic interaction between iron(III) ion and oxygen atom.

The work was supported by the Joint Studies Program (1988-1989) of the Institute for Molecular Science and by the Grant-in-Aid for Scientific Research No. 62470030 from the Ministry of Education, Science and Culture. The authors thank Drs. Kazuyuki Tohji and Yasuo Udagawa of the Institute for Molecular Science for the use of an X-ray absorption spectrometer. Computer calculations were carried out at the computer center of the Institute for Molecular Science in Okazaki.

\section{References}

1. K. L. Cheng, K. Ueno and T. Imamura, "Handbook of Organic Analytical Reagents", CRC Press, Inc., Boca Raton, 1982.

2. Z. Gregorowicz, D. Matysek-Majewska and T. Suwinska, Mikrochim. Acta [Wien], 1985 I, 237.

3. T. Kawashima, T. Minami, M. Kamada and S. Nakano, J. Flow Inject. Anal., 2, 40 (1985).

4. A. E. Martell and R. M. Smith, "Critical Stability Constants", Plenum Press, New York, 1974.

5. A. Avdeef, S. R. Sofen, T. L. Bregante and K. N. Raymond, J. Am. Chem. Soc., 100, 5362 (1978).

6. A. E. Harvey and D. L. Manning, J. Am. Chem. Soc., 72, $4488(1950)$.

7. G. Schwarzebach and A. Willi, Helv. Chim. Acta, 34, 528 (1951).

8. W. A. E. McBryde, Can. J. Chem., 42, 1917 (1964).

9. M. Morin and J. P. Scharff, Anal. Chim. Acta, 60, 101 (1972).

10. M. Magini, J. Inorg. Nucl. Chem., 40, 43 (1978).

11. K. Tohji, Y. Udagawa, T. Kawasaki and K. Masuda, Rev. Sci. Instrum., 54, 1482 (1983).

12. International Tables for X-Ray Crystallography", Vol. III, p. 161, Kynoch Press, Birmingham, 1962.

13. F. J. Harris, Proc. IEEE, 66, 51 (1978).

14. D. E. Sayers, E. A. Stern and F. W. Lytle, Phys. Rev. Lett., 27, 1204 (1971).

15. E. A. Stern, Phys. Rev. B, 10, 3027 (1974).

16. E. A. Stern, D. E. Sayers and F. W. Lytle, Phys. Rev. B, 11, 4836 (1975).

17. B. Lengeler and P. Eisenberger, Phys. Rev. B, 21, 4507 (1980).

18. B.-K. Teo and P. A. Lee, J. Am. Chem. Soc., 101, 2815 (1979).

19. M. Magini, Chem. Phys. Lett., 78, 106 (1981).

(Received March 9, 1990)

(Accepted May 17, 1990) 